Results: Pts reaching clinical remission had significant lower baseline BME sum scores then the non-remission group (mean 1.86 vs. $2.89, p=0.024$ ). At the time of clinical remission 10/45 (22\%) and $11 / 45(24 \%)$ pts had residual talocrural and subtalar synovitis respectively. However, there was no statistically significant difference between patients who relapsed after treatment withdrawal and those who remained in remission concerning synovitis sum scores $(p=0.497)$ as well as BME sum scores $(p=0.741)$ and STI sum scores $(p=0.131)$ at time of clinical remission (table 1).

Abstract FRI0177 - Table 1. BME, synovitis and STI presence in early pSpA pts who relapsed and did not relapse after stopping golimumab therapy

\begin{tabular}{lcc}
\hline & Patients relapsed $(\mathbf{n}=\mathbf{2 0})$ & Patients not relapsed $(\mathbf{n}=\mathbf{2 5})$ \\
\hline BME at baseline & $1.82( \pm 2.29)$ & $1.69( \pm 1.62)$ \\
BME at follow-up & $1.32( \pm 1.12)$ & $1.09( \pm 1.18)$ \\
Change score BME & $0.50( \pm 1.36)$ & $0.52( \pm 1.42)$ \\
Synovitis at baseline & $2.53( \pm 3.11)$ & $3.53( \pm 3.04)$ \\
Synovitis at follow-up & $1.13( \pm 1.41)$ & $1.44( \pm 1.49)$ \\
Change score synovitis & $1.50( \pm 2.71)$ & $2.16( \pm 2.81)$ \\
STI at baseline & $1.67( \pm 1.81)$ & $2.07( \pm 2.38)$ \\
STI at follow-up & $0.93( \pm 0.75)$ & $1.01( \pm 1.12)$ \\
Change score STI & $0.70( \pm 1.34)$ & $1.00( \pm 2.06)$
\end{tabular}

Conclusions: Early pSpA pts who reach clinical remission have less BME on baseline WB MRI compared to those with ongoing disease activity. At time of clinical remission, a substantial part of the participants showed residual ankle synovitis on MRI. However, residual inflammatory lesions detected by WB MRI did not differ significantly between pts who relapsed after treatment withdrawal and those in ongoing clinical remission.

Disclosure of Interest: None declared

DOI: 10.1136/annrheumdis-2018-eular.6365

\section{FRI0179 INTEGRATED LONGITUDINAL ANALYSIS INCREASES PRECISION AND REDUCES BIAS: A COMPARATIVE 5- YEAR ANALYSIS IN THE DESIR COHORT}

A. Sepriano ${ }^{1}$, S. Ramiro ${ }^{1}$, D. van der Heijde ${ }^{1}$, M. Dougados ${ }^{2}$, P. Claudepierre ${ }^{3}$, A. Feydy ${ }^{4}$, M. Reijnierse ${ }^{5}$, D. Loeuille ${ }^{6}$, R. Landewé ${ }^{7} .{ }^{1}$ Rheumatology, Leiden University Medical Centre, Leiden, Netherlands; ${ }^{2}$ Rheumatology, Hôpital Cochin Hôpitaux de Paris, Paris; ${ }^{3}$ Radiology, Hôpital Henri-Mondor, Créteil; ${ }^{4}$ Radiology, Hôpital Cochin, Hôpitaux de Paris, Paris, France; ${ }^{5}$ Radiology, Leiden University Medical Centre, Leiden, Netherlands; ${ }^{6}$ Rheumatology, Hospital Brabois, Nancy, France; ${ }^{7}$ Rheumatology, Amsterdam Rheumatology and Clinical Immunology Center (ARC), Amsterdam, Netherlands

Background: Evaluation of imaging is important in spondyloarthritis $(\mathrm{SpA})$ research, but loss to follow up often jeopardises interpretation of the evaluation. The Interpretation may further be challenged by the fact that often different readers have contributed to scores, in multiple read 'waves'. A common approach is to evaluate patients (pts) with complete follow up (completers analysis), and aggregate scores of individual readers (eg. agreement $>2$ out of 3 readers). These approaches are not assumption-free, may cause non-random data loss, and may as such provide spurious estimates and loss of external validity.

Objectives: We aimed to investigate if the use of all data in an assumption-free manner (a so called 'integrated analysis') affects the precision of estimates for imaging outcomes in pts with axial $\mathrm{SpA}(\mathrm{axSpA})$, with completers analysis as reference standard.

Methods: Pts from the DESIR cohort fulfilling the ASAS axSpA criteria were included. Radiographs and MRIs of the SIJ and spine were obtained at baseline (BL), 1, 2 and 5 years. Each film was scored by 2 or 3 readers in 3 'reading-waves' (wave 1: BL only; wave 2: BL, 1, 2 years; wave 3: BL, 2, 5 years). Each outcome was analysed in two ways: i. according to a 'combination algorithm' ('2 out of 3 ' for binary and mean of 3 readers for continuous variables); and ii. per individual reader. The change of each outcome was analysed by generalised estimating equations (GEE)) with 'time' as explanatory variable. Three analytical approaches were pursued: i) 'integrated-analysis' (including all pts with $\geq 1$ score from $\geq 1$ reader from all waves); ii) completers-only analysis (including only pts with complete 5 year follow-up, using scores from individual readers from wave 3 ); iii) aggregated completers analysis using a combination algorithm (the same as ii but using combined scores).

Results: In total, 413 pts were included (mean (SD) symptom duration: $1.6(0.9)$ years) and 366 completed the 5 year follow up. An analysis with all data from different readers and 'waves' ('integrated analysis') was more inclusive, but did not result in a meaningful loss of precision (width of $95 \% \mathrm{Cls}$ ) of the change-estimates as compared to both completers analyses (table 1). In fact, for low-incident outcomes (e.g.\% of mNY-positive over 5 years), a similar incidence was 'captured', with more precision, by the 'integrated analysis' compared to the completers analysis with combined scores (\% change/year $(95 \% \mathrm{Cl}): 1.1(0.7 ; 1.5)$ vs 1.2 $(0.5 ; 1.8)$, respectively). The same results were seen using continuous outcomes.

Abstract FRI0179 - Table 1. Change per year in the percentage of positive cases for binary imaging outcomes over 5 -years of follow-up, according to 3 different analytical methods, in early axSpA patients fulfilling the ASAS axSpA criteria from the DESIR-cohort

\begin{tabular}{|c|c|c|c|}
\hline & $\begin{array}{l}\text { Integrated } \\
\text { analysis }\end{array}$ & $\begin{array}{c}\text { Completers analysis } \\
\text { with individual } \\
\text { readers scores }\end{array}$ & $\begin{array}{c}\text { Completers } \\
\text { analysis with } \\
\text { combined scores } \\
\text { for readers }\end{array}$ \\
\hline Imaging outcomes & $\begin{array}{l}\% \text { change per } \\
\text { year }(95 \% \mathrm{Cl}) \\
(\mathrm{N}=399-411)\end{array}$ & $\begin{array}{c}\text { \% change } \\
\text { per year }(95 \% \mathrm{Cl}) \\
(\mathrm{N}=342-364)\end{array}$ & $\begin{array}{c}\text { \% change per year } \\
(95 \% \mathrm{Cl}) \\
(\mathrm{N}=338-364)\end{array}$ \\
\hline \multicolumn{4}{|l|}{ SACROILIAC JOINTS } \\
\hline $\begin{array}{l}\text { Sacroilititis on MRI-SIJ (ASAS criteria) } \\
23 \text { fatty lesions on MRI-SIJ }\end{array}$ & $\begin{array}{l}-7.4(-11.7 ;-3.1) \\
4.7(2.7 ; 6.7)\end{array}$ & $\begin{array}{c}-5.4(-8.9 ;-1.9) \\
3.3(1.7 ; 4.9)\end{array}$ & $\begin{array}{l}-3.1(-5.1 ;-1.2) \\
2.1(1.1 ; 3.0)\end{array}$ \\
\hline mNY on X-SIJ (positive/negative) & $1.1(0.7 ; 1.5)$ & $0.9(0.5 ; 1.3)$ & $1.2(0.5 ; 1.8)$ \\
\hline \multicolumn{4}{|l|}{ SPINE } \\
\hline BME: $\geq 3$ lesions on MRI-Spine (ASAS criteria) & $-0.8(-2.3 ; 0.7)$ & $-0.4(-1.4 ; 0.5)$ & $-0.1(-1.2 ; 1.0)$ \\
\hline$\geq 5$ fatty lesions on MRI-Spine & $-0.2(-0.9 ; 0.4)$ & $-0.1(-0.5 ; 0.2)$ & - \\
\hline$\geq 1$ syndesmophyte on $X$-Spine & $0.8(0.5 ; 1.2)$ & $0.5(0.2 ; 0.8)$ & $0.5(0.1 ; 0.9)$ \\
\hline
\end{tabular}

Conclusions: An efficient and entirely assumption-free usage of all data from different readers and 'read-waves' does not compromise precision of the estimates of change in imaging parameters, and may yield increased statistical power for detecting changes with low incidence. In addition, integrated analysis may protect against attrition bias and avoid bias by 'convenient choices'.

Disclosure of Interest: None declared

DOI: 10.1136/annrheumdis-2018-eular.1595

\section{FRI0180 DIAGNOSIS JOURNEY OF PATIENTS WITH ANKYLOSING SPONDYLITIS IN THE UNITED STATES}

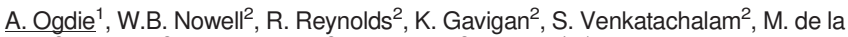
$\mathrm{Cruz}^{3}$, E. Flood ${ }^{3}$, E.J. Schwartz ${ }^{3}$, B. Romero ${ }^{3}$, Y. Park ${ }^{4} .{ }^{1}$ Perelman School of Medicine at the University of Pennsylvania, Philadelphia; ${ }^{2}$ Global Healthy Living Foundation, Upper Nyack; ${ }^{3}$ ICON, Gaithersburg; ${ }^{4}$ Novartis Pharmaceuticals Corporation, East Hanover, USA

Background: A US-based study demonstrated that patients with ankylosing spondylitis (AS) experience a significant delay (on average 14 years) from symptom onset to diagnosis of AS. ${ }^{1}$ Understanding the diagnosis journey of patients with AS and identifying opportunities to reduce misdiagnosis and incorrect referral are crucial to reducing time to diagnosis, preventing irreversible joint damage, and preserving mobility.

Objectives: To describe the patient journey to AS diagnosis from the patient perspective and differences observed between females and males.

Methods: US adults aged $\geq 18$ years with a self-reported diagnosis of AS were recruited through CreakyJoints, an online patient support community comprising patients with arthritis and arthritis-related diseases and their caregivers. Respondents completed a web-based survey on socio-demographics, clinical symptoms, disease burden, and diagnosis history, which included symptoms that led to seek ing care, time from symptom onset to seeking care and from seeking care to AS diagnosis, types of healthcare providers seen, and misdiagnoses. Survey questions were developed following analysis of qualitative interviews of patients with AS and clinical experts, as well as a targeted literature review. Survey results were compared between females and males using 2-sample $t$ tests for continuous variables and chi squared tests for categorical variables.

Results: Among 235 respondents, 174 (74\%) were female. Mean (SD) age of female and male respondents were 48.6 (10.6) and 53.1 (10.3) years, respectively. Although the majority ( $58 \%$ female and $54 \%$ male) sought medical care within the first year of symptom onset, female respondents reported a mean of 17.2 years since first symptom onset and 7.5 years since AS diagnosis; while male respondents reported a mean of 20.0 years since first symptom onset and 11.4 years since AS diagnosis. The most common symptoms that led to seeking medical care were back pain, joint pain, stiffness, and fatigue (figure 1A). During the diagnosis process, patients reported seeking medical care from a general practitioner $(87 \%)$, rheumatologist $(65 \%)$, orthopedist $(27 \%)$, chiropractor $(26 \%)$ and urgent care/emergency room doctor $(21 \%)$ with no differences between females and males. The most commonly reported misdiagnoses were back problems $(56 \%)$, psychosomatic $(23 \%)$, and sciatica $(21 \%)$ in males, while psychosomatic $(41 \%)$, back problems $(40 \%)$, and anxiety/depression $(24 \%)$ were most common in females. Significantly higher proportions of females reported misdiag noses of fibromyalgia ( $21 \%$ vs $7 \%$ ) and psychosomatic ( $41 \%$ vs $23 \%$ ) (figure 1 B). 